\title{
El Proyecto Manubuild: una propuesta de la aplicación de sistemas industrializados a la vivienda colectiva en España
}

\section{The Manubuild Project: a proposal of the application of industrialized systems in the collective housing in Spain}

C. Ruiz-Larrea*, E. Prieto*, A. Gómez", H. Bugueño**

\section{RESUMEN}

En marzo de 2006, fue convocado por la Empresa Municipal de la Vivienda y el Suelo de Madrid (EMVS) un concurso restringido de ideas arquitectónicas como primer paso para la construcción de un edificio, en el contexto de la estrategia marcada por el Proyecto Integrado I+D+i Manubuild, con financiación de la Comisión Europea a través del $6^{\circ}$ Programa Marco.

El objetivo de este concurso, en el que participaron diferentes equipos europeos, fue obtener propuestas de alto nivel arquitectónico sobre nuevos tipos edificatorios -en el ámbito de la climatología y la cultura mediterráneos-, con aplicación de sistemas constructivos industrializados, abiertos y sostenibles, de tal modo que las ideas seleccionadas constituyesen el punto de partida conceptual para el edificio que la EMVS tenía previsto realizar en Madrid como demostrador de la investigación.

La propuesta ganadora -redactada por el Estudio RLA- pretende superar las inercias que tradicionalmente han lastrado a la construcción en España, un modelo pseudoartesanal fundado en la promoción inmobiliaria oportunista, con el resultado de que nuestro modo de crecer ha sido el más insostenible de la Unión Europea.

La estrategia del proyecto rehúye asimismo cualquier industrialización o seriación cerrada, optando por un planteamiento intelectual que interpreta las ventajas de la industrialización en clave energética, proponiendo una respuesta integral que dé cuenta de nuevos tipos edificatorios flexibles y con capacidad para evolucionar,

\section{SUMMARY}

In March 2006 the Madrid Municipal Housing and Property Corporation (Spanish initials, EMVS) announced a limited competition of architectural ideas as the first step in erecting a building designed to the strategy laid down in the Integrated Manubuild R\&D+i Project, funded under the European Commission's $6^{\text {th }}$ Framework Programme.

The purpose of this competition, in which a number of European teams participated, was to obtain high-calibre architectural proposals for new building types for the Mediterranean climate and culture, using open and sustainable industrialized construction systems. The ideas selected were to constitute a conceptual springboard for a building that the EMVS planned to erect in Madrid as the product of the research.

The winning proposal, authored by Estudio RLA, aims to break the inertia that has traditionally weighed on Spanish construction, a model based on pseudo-craftsmanship and opportunistic property development that generated the least sustainable growth pattern in the European Union.

The project strategy rules out closed industrialization or serial production, opting for an approach in which the advantages of industrialization are interpreted in the context of energy savings. The proposal would be for comprehensive design in line with new, flexible building types able to evolve and adapt to users' changing needs while
Informes de la Construcción Vol. 61, 513, 47-58, enero-marzo 2009 ISSN: 0020-0883 elSSN: 1988-3234 doi: 10.3989/ic.08.039

\footnotetext{
* RLA RuizLarrea\&Asociados (Madrid, España)

${ }^{* *}$ Colaborador (Dpto. I+D+i de RLA).
} 
adaptándose a las necesidades cambiantes de los usuarios y asegurando la compatibilidad geométrica que los sistemas industrializados exigen.

La flexibilidad espacial requerida por las bases del concurso se desarrolla, de este modo, con sistemas industrializados que generan la estructura portante, las instalaciones, los sistemas bioclimáticos pasivos y activos y el sistema de la envolvente, permitiendo el alcanzar una solución completamente abierta donde es posible combinar los elementos constructivos dentro de una gama estandarizada de productos y proveedores.

195-11

Palabras clave: industrialización, sostenibilidad, Manubuild, catálogos abiertos.

\section{ANTECEDENTES}

En marzo de 2006, fue convocado por la Empresa Municipal de la Vivienda y el Suelo de Madrid (EMVS) un concurso restringido de ideas arquitectónicas como primer paso para la construcción de un edificio demostrador, en el contexto de la estrategia marcada por el Proyecto Integrado I+D+i Manubuild, con financiación de la Comisión Europea a través del $6^{\circ}$ Programa Marco.

El objetivo de este concurso, en el que participaron diferentes equipos europeos, fue obtener propuestas de alto nivel arquitectónico sobre nuevos tipos edificatorios -en el ámbito de la climatología y la cultura mediterráneos-, con aplicación de sistemas constructivos industrializados, abiertos y sostenibles, de tal modo que las ideas seleccionadas constituyesen el punto de partida conceptual para el edificio que la EMVS tenía previsto realizar en Madrid como demostrador de la investigación.

Este concurso, fallado en abril de 2006, fue ganado por el equipo Piercy Conner Architects.

A finales de 2006, se convocó un segundo concurso, también restringido, en el que se invitaba a un grupo de equipos españoles de reconocido prestigio, con el objeto de adjudicar el proyecto definitivo y la dirección de obra del edificio de 50 viviendas de protección pública en el PAU de Carabanchel. El objetivo buscado por la EMVS era "construir un edificio experimental que actúe como demostrador de la operatividad y la eficacia de las nuevas soluciones constructivas. Este edificio deberá ser altamente industrializado, sostenible, flexible, personalizado para el usuario, más económico, más seguro y de menos coste en su mantenimiento que las ensuring the geometric compatibility required by industrialized systems.

The spatial flexibility required in the competition rules is, then, embodied in industrialized systems that generate the bearing structure, services, active and passive bio-climatic systems and the envelope. This provides for a wholly open solution in which building elements selected from a range of standardized products and suppliers can be combined.

Keywords: industrialization, sustainability, Manubuild, open catalogues.

construcciones convencionales y estar abierto, además, a las innovaciones".

Este segundo y definitivo concurso fue fallado en enero de 2007 y ganado por el equipo RLA (Ruiz-Larrea\&Asociados).

\section{2. "TRES AL CUBO". EL PROYECTO}

\subsection{El proyecto en el contexto actual de la industrialización en España}

La propuesta pretende superar las inercias que tradicionalmente han lastrado a la construcción en España. Este modelo ha estado determinado últimamente por un cómodo desarrollo cuantitativo fundado en la promoción inmobiliaria oportunista, con el resultado de que nuestro modo de crecer ha sido el más insostenible de la Unión Europea. Sin embargo, lo más preocupante es que junto a esta situación coyuntural pervive el problema -ya crónico- de la falta de especialización del sector, dependiente en exceso de una mano de obra poco cualificada, de tal manera que resulta casi imposible consolidar cualquier cultura de la innovación.

Este modelo técnicamente pseudoartesanal -que hereda lo peor de los sistemas tradicionales habiendo perdido por el camino lo mejor de ellos (su especialización y alta calidad)- y económicamente oportunista, contribuye a consolidar, además, tipos edificatorios anacrónicos que no responden a los nuevos modos de habitar y hacer ciudad que reclaman las sociedades contemporáneas.

Sin embargo, consideramos que la respuesta a estas demandas no puede llevarse a cabo utilizando las tradicionales herramientas de la industrialización cerrada, un modelo que conduce a productos homogéneos y estanda- 
rizados y que tan infructífero se ha demostrado hasta el momento para la arquitectura y la sociedad. El problema de la construcción no puede abordarse exclusivamente en términos de seriación. Por el contrario, los modos se producción hace mucho que abandonaron el modelo histórico de la cadena de montaje completa -el modelo General Motors- por el abierto de ensamblaje de componentes -el modelo Toyota-. Un sistema industrializado eficaz es siempre abierto y puede adaptarse a cualquier (buena) arquitectura. El reto de ésta, por lo tanto, no consiste en mimetizarse en un producto industrial acabado sino en construirse utilizando verdaderos procesos de ensamblaje de los componentes de un catálogo siempre abierto a soluciones innovadoras.

Estos catálogos deben ser flexibles de modo que puedan dar respuesta a cualquier arquitectura. Pero, para que esto sea posible, el proyecto arquitectónico debe redactarse en términos homologables. La forma debe contener en su interior un lenguaje cifrado de mínimos que permita que la arquitectura sea compatible con los catálogos de productos industrializados (de igual modo que existe un lenguaje de compatibilidades y tolerancias en el montaje de la producción industrial por componentes). No basta con aplicar los sistemas industrializados a la geometría; por el contrario, el reto consiste precisamente en generar esta geometría a partir de un modo de trabajar transversal.

La estrategia del concurso hereda este planteamiento intelectual e incorpora un modo de trabajar que propone nuevos tipos edificatorios flexibles y con capacidad de evolucionar, adaptándose no sólo a las necesidades cambiantes de los usuarios sino a los requisitos de compatibilidad que los sistemas industrializados abiertos exigen $y$ completando y perfeccionando a éstos con nuevos productos que permitan hacer frente a la demanda cada vez más creciente de ahorro energético.

La flexibilidad espacial requerida por las bases del concurso se desarrolla, de este modo, con sistemas industrializados que generan la estructura portante, las instalaciones, los sistemas bioclimáticos pasivos y activos y el sistema de la envolvente, permitiendo el alcanzar una solución completamente abierta donde es posible combinar los elementos constructivos dentro de una gama estandarizada de productos y proveedores.

\subsection{El "grado cero" de la industrialización: modulación, geometría}

¿Cómo compatibilizar las medidas y tolerancias de los catálogos industriales con la flexibilidad que requieren las viviendas contemporáneas? Inevitablemente, la respuesta a esta pregunta se escribe en términos de geometría. La primera decisión de la arquitectura es, en estos casos, también inevitablemente convencional y consiste en la adopción de un módulo métrico -un alfabeto elemental- capaz de permitir que la geometría esté abierta no sólo durante el proceso proyectual sino también durante la vida útil del edificio.

Adoptamos, así, el tradicional módulo de $60 \mathrm{~cm}$ para configurar una malla geométrica universal, a modo de casillero donde colocar el resto de las piezas. La suma de 5 módulos elementales conduce, a su vez, a un módulo de un orden superior, un cubo de $3 \mathrm{~m}$ en sus tres dimensiones - "Tres al cubo"-. Los espacios habitables pueden entenderse, de este modo, como una combinación de diferentes módulos o células especializadas para responder a los requerimientos funcionales o energéticos exigidos a cada una de ellas. La matriz geométrica cúbica permite, así, diferentes configuraciones formales adaptables a cualquier área de movimiento y un crecimiento ilimitado en altura. La propuesta, por lo tanto, parte de una matriz geométrica completamente abierta.

Resolver satisfactoriamente esta combinatoria implicó una estrategia capaz de encarar un alto nivel de complejidad. Sin embargo, la estructura matricial arriba propuesta permite descomponer esta complejidad en diferentes niveles para responder específicamente a cada uno de ellos de la mejor manera posible.

\footnotetext{
1.- La matriz "Tres-al-cubo" es una estructura completamente abierta que permite hacer compatibles los catálogos de productos industrializados con la geometría de la vivienda. Esta matriz permite diferentes configuraciones formales adaptables a cualquier área de movimiento horizontal o vertical.
}
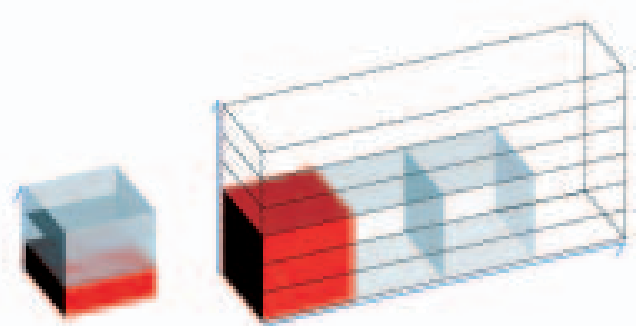

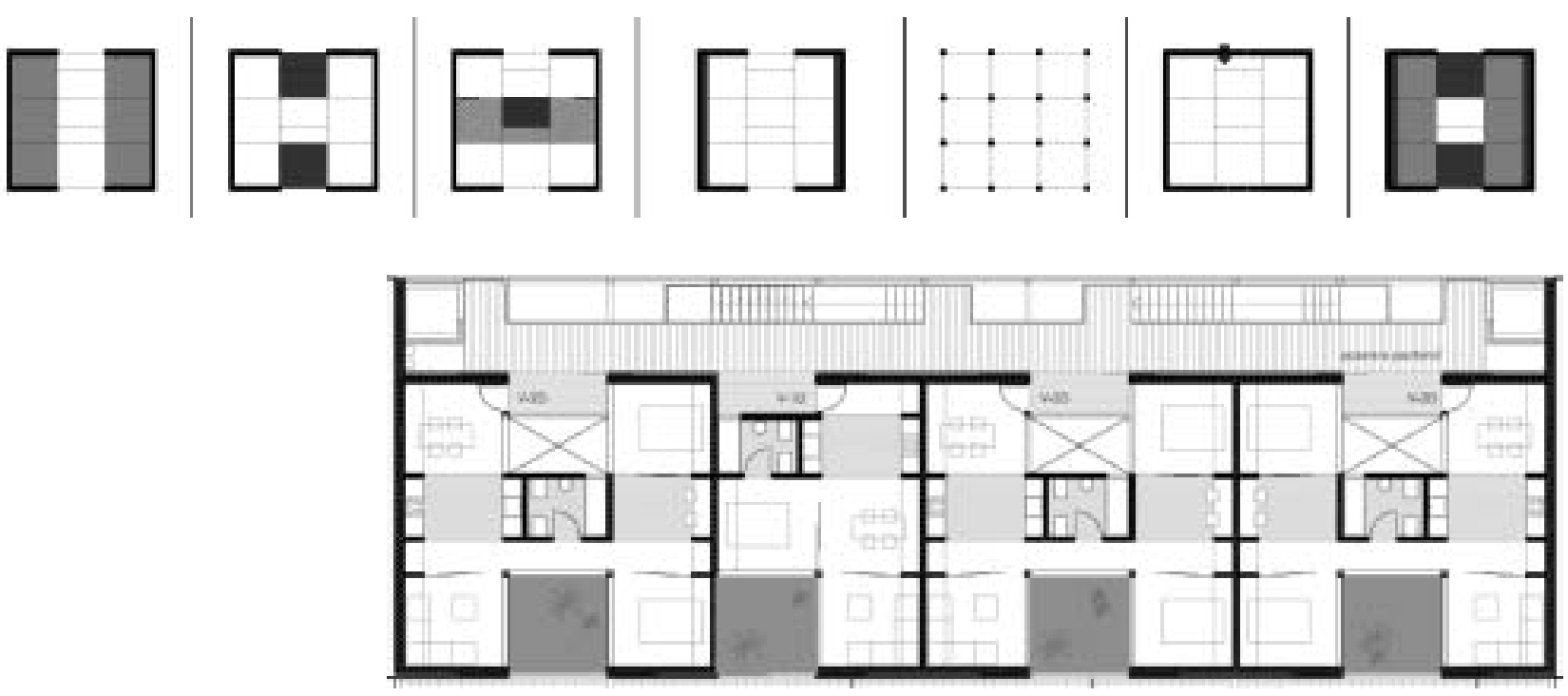

2.- La estructura matricial propuesta permite descomponer la complejidad del programa en diferentes niveles para responder específicamente a cada uno de ellos de la mejor manera posible. El tipo edificatorio, de este modo, es el resultado final de combinar los diferentes módulos o células.

3.- Al situarse las células técnicas y bioclimáticas en posiciones técnicas, los espacios vivideros están servidos homogéneamente de modo que es posible que éstos adopten configuraciones de gran versatilidad.
Los tres tipos edificatorios desarrollados en el proyecto son el resultado final de combinar los diferentes módulos o células. Con el fin de alcanzar la máxima flexibilidad en las distribuciones, se diseñaron dos espacios abiertos situados a lo largo de las dos alas del tipo. Las células orientadas hacia las fachadas Sur y Norte están ocupadas, por su parte, por dos espacios bioclimáticos que aprovechan la diferencia de potencial energético entre ambas orientaciones. La célula central está asignada a un módulo técnico que canaliza la energía desde y hacia los espacios vivideros. Esta organización permite adaptar la retícula estructural a la matriz geométrica. El intervalo de 3 m que define a ésta es adoptado por aquélla de tal modo que se reduzcan al máximo las luces estructurales y sea posible aplicar un sistema completamente industrializado de pilares y jácenas homogéneas conectados por nudos especializados y formando una red isotrópica que no interfiere en los espacios habitables.

Los espacios de almacenamiento, finalmente, se sitúan en las franjas de medianería de tal modo que tampoco interfieran con los espacios habitables.

\subsection{Flexibilidad tipológica}

Esta estrategia aditiva o combinatoria de módulos especializados compatibles con los catálogos industrializados permite un máximo de flexibilidad en la configuración interna de los espacios de la vivienda. Como las células técnicas y bioclimáticas están ubicadas en la parte central de la vivienda, pueden servir homogéneamente al resto de las células vivideras, las configuraciones que puedan adoptar éstas son prácticamente ilimitadas y, por lo tanto, las viviendas pueden personalizarse.

El sistema permite, por ejemplo, no sólo una disposición de uno o dos dormitorios para familias convencionales o una solución de tres dormitorios para estudiantes sino también el combinar vivienda y trabajo en la misma vivienda o incluso, si se almacenan los muebles en el perímetro, usar todo el espacio vividero para una reunión o una fiesta.
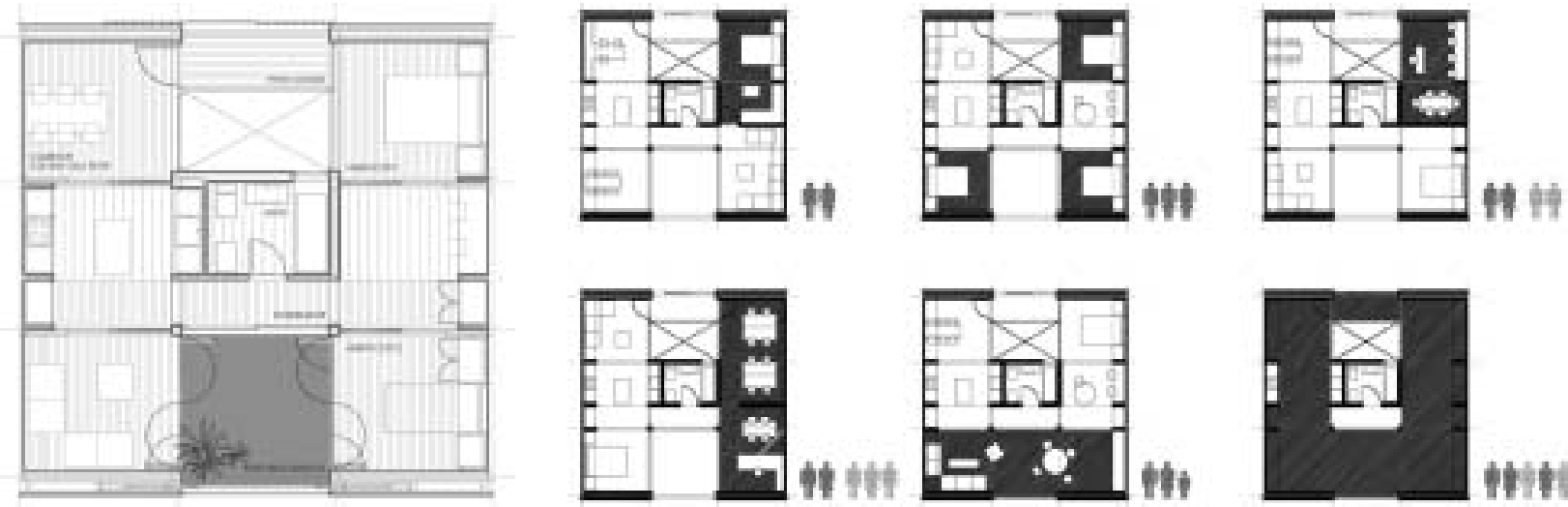
La organización del espacio interior de la vivienda es, por lo tanto, geométricamente muy sencilla pero muy rica en cuanto a su capacidad adaptativa. Los espacios vivideros se disponen en torno a las células bioclimáticas y el módulo técnico. Todos los paneles que forman las particiones interiores son móviles y escamoteables para modificar la superficie de las distintas áreas de acuerdos a las necesidades cambiantes de los usuarios (adaptables a un convencional ritmo díanoche o bien al números de miembros de la familia, etc.).

La combinatoria surgida de la modulación permite también, como ya hemos avanzado, que la matriz geométrica pueda adaptarse a las áreas de movimiento de diferentes situaciones urbanísticas. En el caso del proyecto Manubuild el programa se distribuyó en dos bloques definidos por una geometría sencilla que sitúa los corredores de comunicación en la orientación Norte liberando, de este modo, la fachada opuesta de tal modo que pueda aprovecharse al máximo el potencial energético de la misma. Estos criterios bioclimáticos definieron, de igual modo, la posición y altura relativa de los dos bloques a fin de evitar la obstrucción solar entre los mismos.

\subsection{Industrialización de la estructura portante}

\section{Estructura portante primaria}

En la elección del tipo estructural más idóneo, se tuvieron en cuenta las condiciones geométricas del proyecto, los obvios requerimientos de cálculo (acciones gravitatorias, sobrecargas de uso, asunción de empujes horizontales, etc.) y, sobre todo, su adecuación a las peculiaridades funcionales y técnicas de la propuesta y a la decidida apuesta por la modulación y la facilidad constructiva.

Bajo rasante, el concurso planteaba una estructura de muros, cimentaciones y losas de hormigón armado prefabricado. Es la gama de materiales idóneos por su buena relación con el terreno y sus condiciones de subterraneidad. A partir de la cota y a través de una losa estructural de transición, la estructura sale al aire y por ello se impone su condición de ligereza. Por ello, se propuso construir la estructura sobre rasante por elementos metálicos.

Junto a estas condiciones de ligereza y rapidez constructiva, la estructura de acero permitía una compatibilidad inmediata con la matriz cúbica del proyecto. Así, la trama geométrica básica de $3 \times 3$ m conduce a luces estructurales muy reducidas lo que permitía que las secciones aparentes de los elementos portantes verticales (pilares) y horizontales
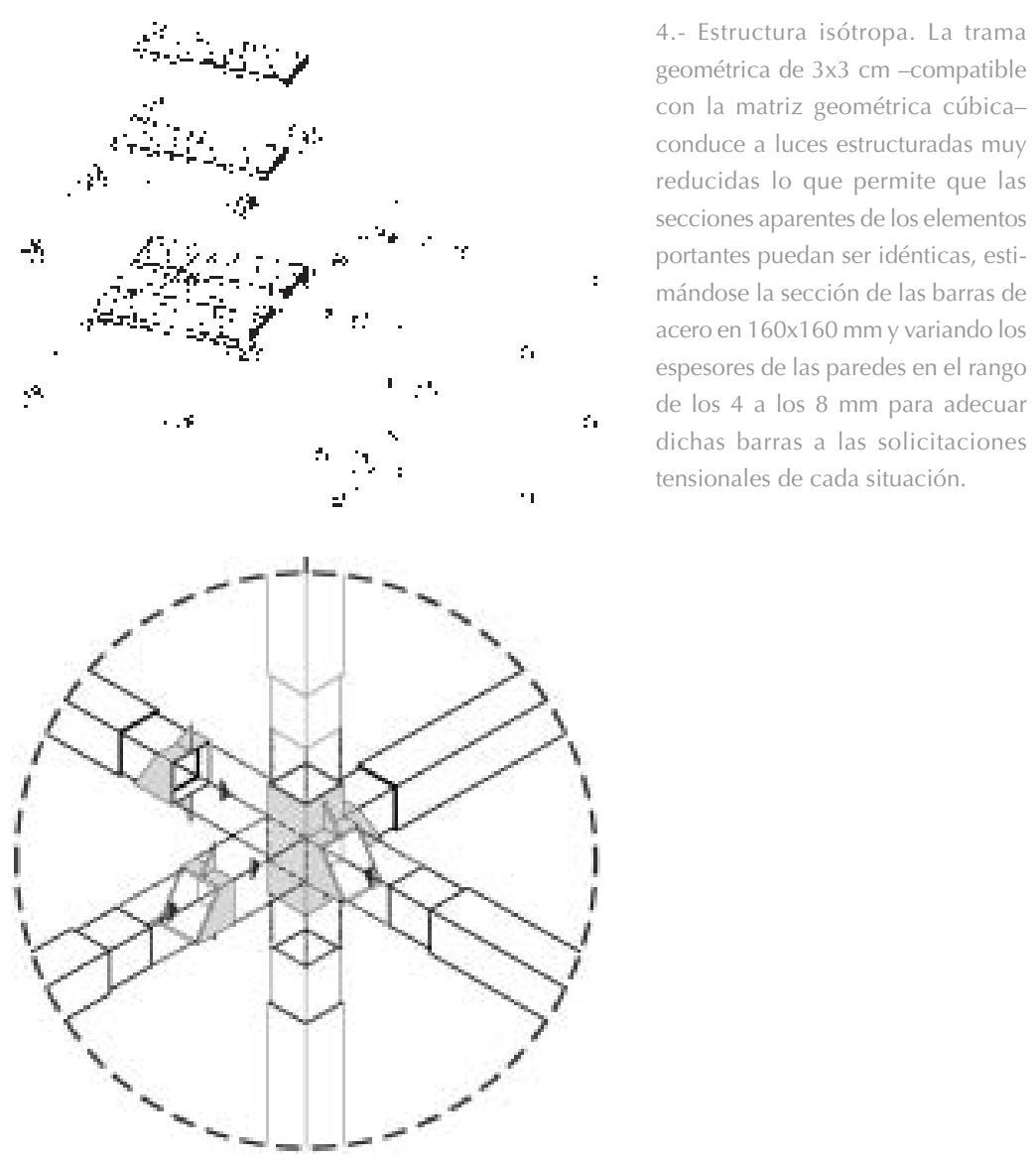

(vigas, zancas, etc.) pudieran ser idénticas, estimándose su sección en 160×160 mm para un tubo de acero y variando los espesores de las paredes del mismo en el rango de los 4 a $8 \mathrm{~mm}$ para adecuar las barras a las solicitaciones tensionales de cada situación. La estabilidad horizontal se aseguraba mediante un orden secundario de barras de arriostramiento en cruz, situados en algunos planos interiores o de fachada que no presentasen huecos.

La transición entre las estructuras bajo y sobre rasante, de hormigón armado y acero, se resolvía en el concurso con una losa bidireccional de hormigón armado del suficiente espesor para hacer frente a las solicitaciones derivadas de los apeos. Esto es así por la conveniencia de ser diferentes las tramas inferior $(3 \times 3 \mathrm{~m})$ con la inferior $(7,2 \times 7,2 \mathrm{~m})$, idónea para la perfecta distribución de las plazas de garaje en las plantas inferiores.

\section{El "Nudo-build"}

La voluntad de obtener la máxima industrialización en la estructura sobre rasante se concreta en plantearla de forma que, si se estima necesario, pueda construirse completamente atornillada, sin excluir tampoco la posibilidad de ser parcial o totalmente soldada. EI corazón de cada uno de los nudos de la red 
5.- Estructura de hormigón pretensado. La estructura de hormigón consiste en dos pórticos formados por pilares y vigas completamente prefabricados en los cuales se apoya la estructura horizontal constituida por placas alveolares de $12 \mathrm{~m}$ de luz. El sistema de rigidización dev los nudos formados por varillas roscadas de acero de diferentes diámetros y recibidas a los pilares mediante resinas epoxi (sistema BOMA-PUJOL).

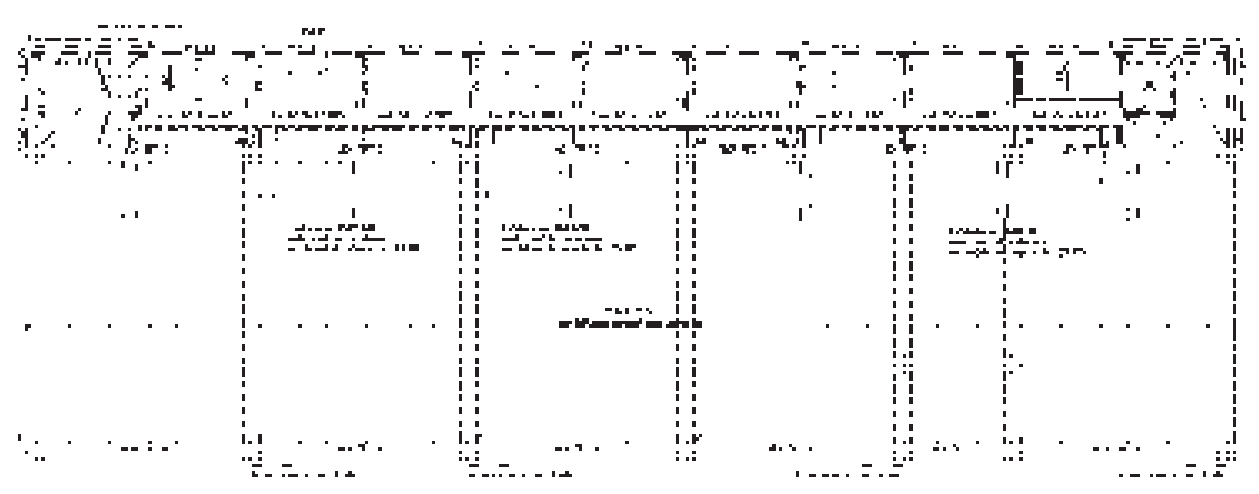

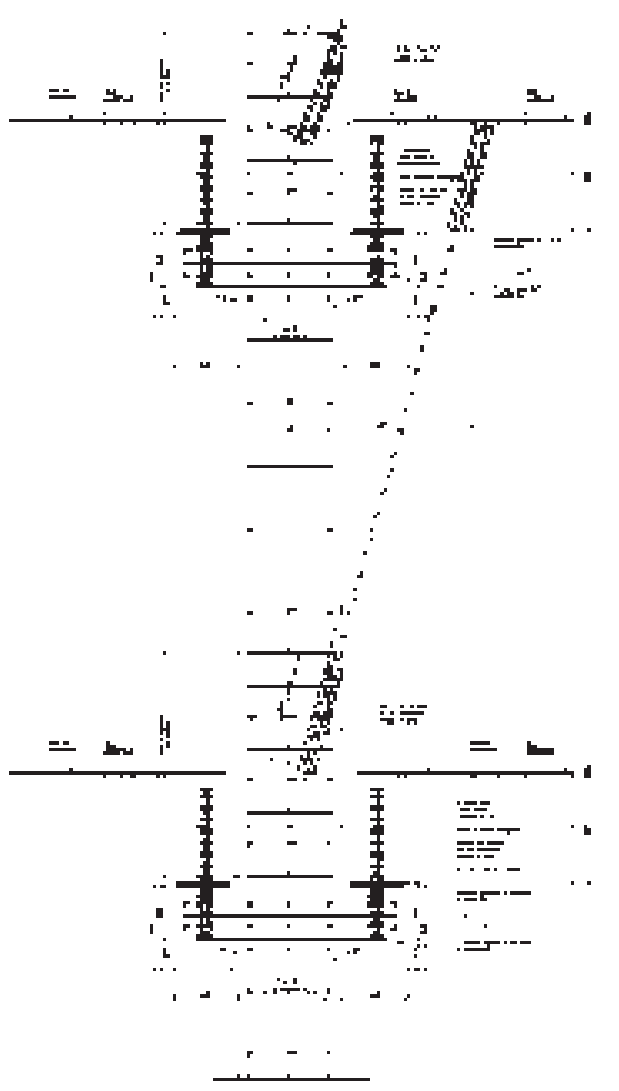

estructural isótropa y cada una de las barras que acometen al mismo, serían fabricados en taller para ser ensamblados con rapidez y precisión en obra.

\section{Estructura horizontal}

La estructura horizontal, soportada en general en tramos de $3 \times 3 \mathrm{~m}$ se planteaba con elementos unidireccionales que aprovecharían en numerosas ocasiones sus posibilidades de hiperestaticidad en su condición de banda continua. Para conseguir este efecto, se planteaban apoyadas sobre la estructura principal de barras de tubo cuadrado.

A partir de estas condiciones de contorno, era posible resolver la estructura horizontal de diferentes maneras, pudiendo estar constituidas por chapas grecadas colaborantes o no (en función de la capacidad de aislamiento ígneo de los falsos techos), con su correspondiente capa de compresión de hormigón armado, o bien resolverla con placas alveolares pretensadas prefabricadas en aquellas zonas donde esta solución presente ventajas constructivas o minimice sensiblemente los plazos de ejecución.

Dificultades durante la fase de proyecto y modificaciones del planteamiento estructural

El interés del Programa Manubuild en comparar soluciones estructurales de diferentes materiales, así como los importantes problemas en la asignación presupuestaria desde el origen del proyecto condujeron a que, en el proyecto final, la idea de una estructura isótropa de barras de acero tuviese que ser modificada, optándose por una estructura de hormigón pretensado prefabricado para el bloque Sur y una estructura industrializada de acero de luces amplias para el bloque Norte.

El esquema de la estructura de hormigón -desarrollada con el asesoramiento de Prefabricados Pujol y calculada por BOMA- es sencillo y está orientado a reducir el número de elementos portantes de modo que pueda atenuarse cualquier servidumbre estructural que entre en conflicto con la flexibilidad espacial buscada. Consiste en dos pórticos formado por pilares y vigas completamente prefabricados en los cuales apoya la estructura horizontal constituida por placas alveolares de $12 \mathrm{~m}$ de luz. El sistema se rigidización de los nudos está formado por varillas roscadas de acero de diferentes diámetros y recibidas a los pilares mediante resinas epoxi, de tal modo que, al no tenerse que utilizar las soluciones habituales basadas en la soldadura o el homigonado in situ, se simplifica y abarata la ejecución de los nudos. El arriostramiento de los nudos se complementa, asimismo, por la rigidez que proporciona al conjunto estructural el entramado de perfiles de acero de la fachada Norte. 
El esquema de la estructura de acero -calculada por NB35- es geométricamente análogo al anterior y consiste, asimismo, en dos pórticos paralelos formados por vigas y pilares de acero sobre los que apoyan placas alveolares de hormigón pretensado. Todas los elementos metálicos son de gran formato y se traen conformados directamente desde taller de modo que en obra tan sólo tengan que ejecutarse las juntas bien con soluciones electrosoldadas bien roblonadas, dependiendo del tipo de unión.

\subsection{Industrialización de las instalaciones}

El esquema geométrico que define al tipo edificatorio hace posible, como hemos visto arriba, el concentrar las instalaciones de la vivienda, en un módulo técnico. Este módulo técnico está formado, a su vez, por distintos componentes especializados: el módulo de aseo prefabricado, el kit de reciclaje de aguas, el módulo de enfriamiento evaporativo y el patinillo vertical industrializado.

\section{Aseos prefabricados}

Los aseos son completamente prefabricados y se colocan en obra, directamente traídos desde la fábrica, con los acabados finales, que pueden personalizarse dependiendo del usuario. La solución constructiva de estos aseos -desarrollados por MARTIFER, una empresa portuguesa con larga experiencia en prefabricación-consiste en un entramado de acero galvanizado revestido por tableros ligeros fenólicos o de composite de yeso que admiten prácticamente cualquier acabado superficial.

Entre las ventajas implicadas, en este caso, por la prefabricación, se encuentra la posibilidad de asegurar la correcta ejecución de las juntas entre los conductos de acometida y de salida de los aparatos sanitarios, un problema mal resuelto en la construcción pseudoartesanal. Asimismo, se diseñó el módulo de aseos de tal modo que permitiese la flexibilidad de adoptar prácticamente cualquier disposición de los sanitarios al dejarse registrables las juntas de conexión de las tuberías.

\section{Kit de reciclaje de aguas}

El reciclado completo de las aguas pluviales, Ilevado a cabo por un minicentral conectada a un aljibe que recupera el agua depositada en las cubiertas y zonas urbanizadas de la parcela, se complementó en el concurso con un sistema individual de reciclaje de las aguas grises provenientes del lavabo y la ducha para ser reutilizadas en la cisterna del WC, la lavadora, la limpieza y el riego, lográndose un ahorro real del $30 \%$ en el consumo de agua. Sin embargo, la anticuada normativa
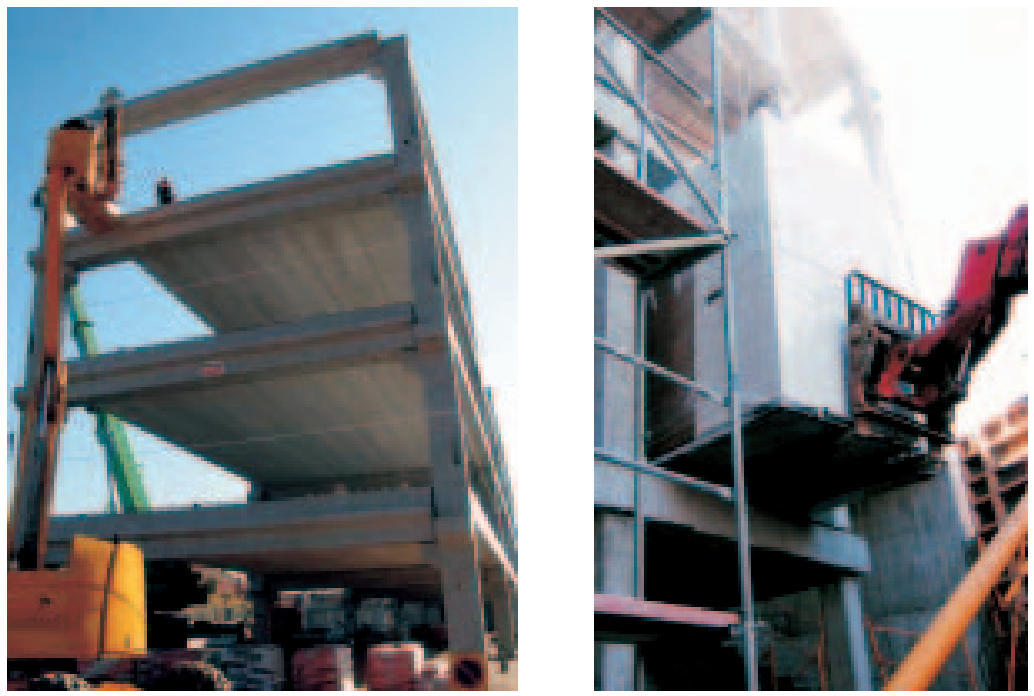

de salubridad que, paradójicamente, es cada vez más restrictiva en el reciclaje de aguas, impidió la aplicación de esta medida en el proyecto definitivo.

\section{Módulo de enfriamiento evaporativo}

El problema, ya clásico, de la integración de los aparatos de climatización en la vivienda, se resolvió en el Proyecto Manubuild mediante la integración en la célula técnica de un módulo de enfriamiento evaporativo por agua (sistema adiabático) formado por un ventilador eléctrico, un filtro y una rejilla de impulsión constituido por un entramado con forma de panel de colmena de fibra de vidrio que se humidifica y a través de la cual pasa el aire filtrado e impulsado por el ventilador.

El sistema se completa con una pieza que permite la recuperación y el saneamiento del agua sobrante y con un filtro especial que evita cualquier problema de salubridad del aire susceptible de ser causado por un exceso de humedad.

Es importante destacar que este sistema, que trabaja con un reducido consumo eléctrico pero genera un considerable gasto de agua, debe ser siempre complementario a una estrategia integrada de soluciones pasivas y debe ser, por lo tanto, aplicado exclusivamente para aportar una bajada de temperatura en los periodos más calurosos y, en el caso de Madrid, secos del año.

\section{Patinillo vertical industrializado ("Service Core")}

El Service Core o patinillo vertical industrializado, desarrollado por Dragados en el contexto del Programa Manubuild, está concebido para integrar todos los conductos y servicios relacionados con las instalaciones de cada vivienda.

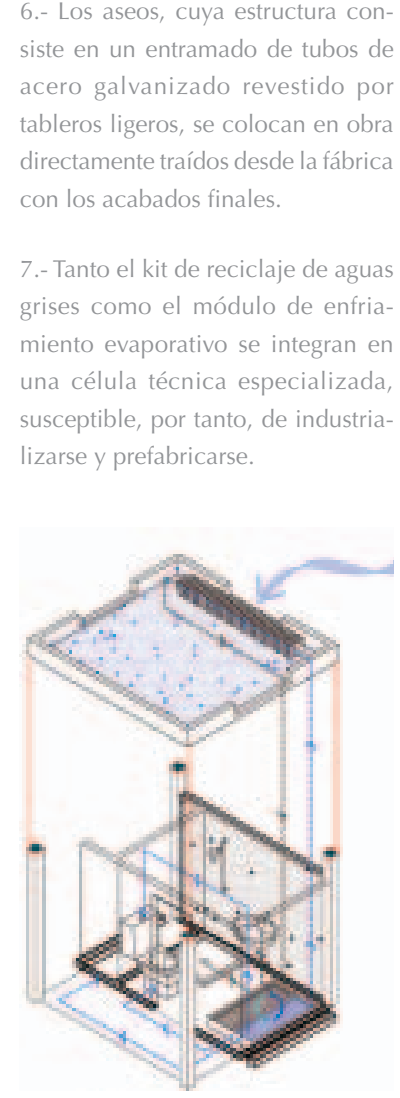


8.- El "Service core" o patinillo vertical industrializado, desarrollado por Dragados en el contexto del programa Manubuild, está concebido para integrar todos los conductos y servicioos relacionados con las instalaciones de la vivienda.

9.- La geometría de la parcela permite una disposición que llega a optimizar el aprovechamiento de la energía solar. Esta disposición hace posible, asimismo, una ventilación natural óptima al generar viviendas pasantes.
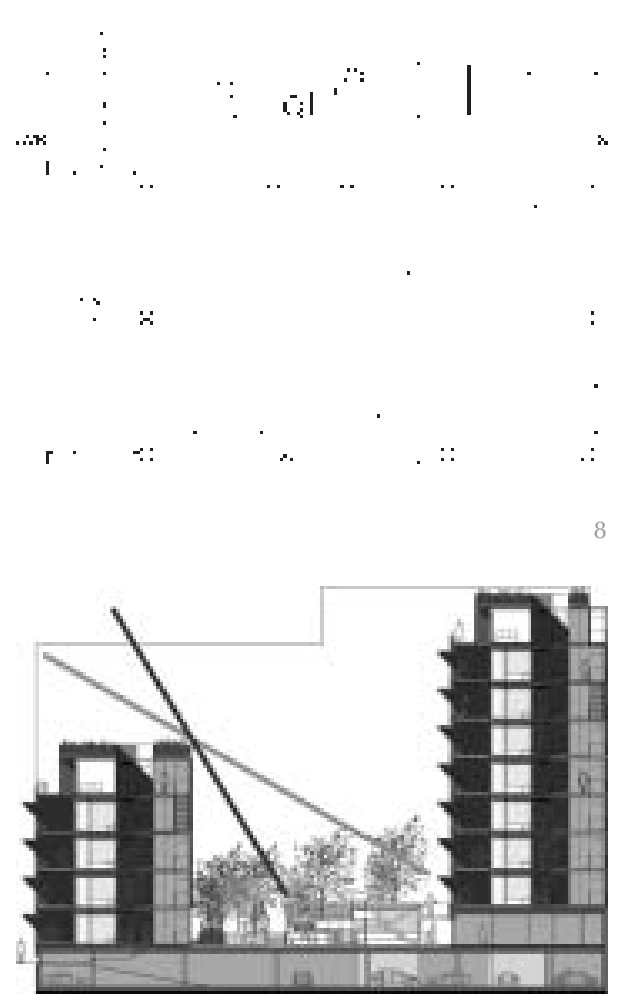

9

El proyecto en Carabanchel el patinillo se sitúa en posición tangencial a la célula técnica de tal modo que sea posible una conexión limpia, eficaz y, sobre todo, registrable entre los conductos procedentes del interior de la vivienda y los conductos verticales.

Dadas, además, las especiales características del proyecto en cuanto a la aplicación de sistemas bioclimáticos, el patinillo industrializado incluye no sólo los conductos convencionales -bajantes, ventilación- sino otros especializados -chimeneas solares, bocas de captación para la renovación natural del aire de las viviendas-, integradas en una solución completamente prefabricada y desarrollada en origen en una cadena de montaje robotizada.

\section{6. "Industrialización" energética: sistemas pasivos y activos}

El tipo edificatorio organiza los espacios vivideros en torno a dos células especializadas situadas con orientaciones opuestas (Sur y Norte, respectivamente) de tal modo que pueda aprovecharse la diferencia de potencial energético generada por esta situación a lo largo del régimen climático anual.

Estos espacios bioclimáticos, por lo tanto, intercambian energía dependiendo de la época del año y constituyen el ejemplo más eficaz en la vivienda de una estrategia de ahorro energético pasivo.

\section{Condiciones de soleamiento y ventilación}

La geometría de la parcela permite una disposición que optimiza el aprovechamiento de la energía solar. Se dispusieron, como hemos visto más arriba, dos bloques de diferente altura orientados al Sur. Esta decisión implicó un cambio en la geometría de las áreas de movimiento, pues la normativa obligaba a situar los bloques con orientaciones muy desfavorables para el clima de Madrid (Este y Oeste).

Gracias a estas medidas, las zonas vivideras pudieron orientarse hacia el Sur, destinándose, por el contrario, la Norte a los vestíbulos, corredores y zonas de circulación. Esta disposición permitía, asimismo, una ventilación óptima al generar viviendas pasantes.

\section{Caldera solar}

Los espacios vivideros de las viviendas están dispuestos en torno a células orientadas al Sur que, a modo de galería acristalada, funcionan durante el invierno como verdaderas calderas solares. Esta galería está cerrada por un doble cerramiento de vidrio que captura, por efecto invernadero, la radiación incidente durante los días soleados de invierno, hasta elevar la temperatura interna del aire al entorno de $\operatorname{los} 20^{\circ} \mathrm{C}$.

Este aire precalentado se distribuye de manera homogénea a los espacios vivideros y, asimismo, se conecta a los radiadores a través de conductos de chapa que, empotrados en el suelo de la galería solar, aprovechar la inercia térmica del forjado para subir la temperatura del aire de renovación. Para evitar el sobrecalentamiento de la galería durante el verano, se han diseñado elementos de protección solar que rechazan la radiación directa (parasol horizontal y screen microperforado vertical con acabados reflectantes).

Sistemas de renovación y refrigeración de aire

La renovación del aire de los espacios vivideros es necesaria para asegurar unos buenos niveles de confort, pero inevitablemente trae aparejada el problema del puente térmico. Para hacer frente a esta situación -fomentada por el actual CTE-, durante el invierno, como hemos visto más arriba, el aire externo de renovación penetra a través de conductos empotrados en el suelo de la galería solar y pasa, después, a los radiadores, de tal modo que el aire no se introduzca nunca de manera directa al interior de la vivienda.

Durante el verano, la galería solar se cierra y se protege de la radiación. Durante este periodo, el aire de renovación entra a través 


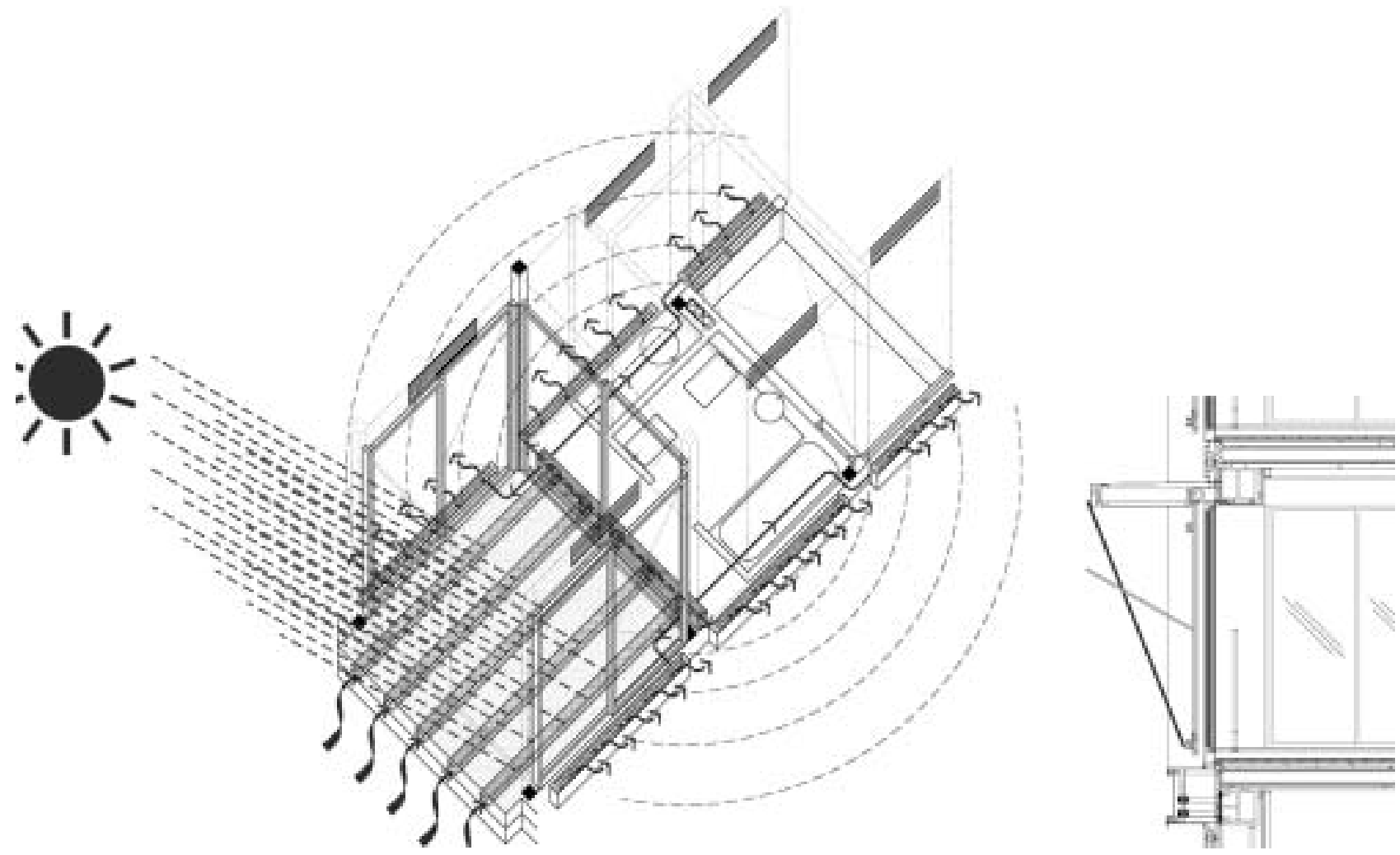

de los patios orientados a la fachada Norte, (totes C)

Temperatures and Loads siempre en sombra y enfriados mediante la evapo-transpiración debida a la vegetación allí plantada. Si es necesario, este aire pasa, después, a la máquina adiabática, con lo que se completa la refrigeración hasta alcanzar una temperatura de confort propuesta en $25^{\circ} \mathrm{C}$.

La renovación del aire se completa con dos chimeneas solares integradas en la célula técnica que contribuyen al tiro natural del sistema. Estas estrategias se combinan con otras que aprovechan el tipo pasante para favorecer la ventilación nocturna, un efecto especialmente acusado en la climatología de Madrid con el cual pueden obtenerse hasta un $30 \%$ de ahorro estimado en la refrigeración de las viviendas.

\section{El envolvente "Biopix"}

El altísimo desarrollo de la técnica contemporánea permite abrir nuevos caminos a la investigación de las pieles de edificación. Sin duda, entre las nuevas posibilidades, las envolventes activas constituyen un campo no explorado de gran importancia. No sólo es lógico el pensar que las envolventes pueden ser rigurosas protecciones pasivas frente al exterior sino que, por primera vez, pueden convertirse en epidermis generadoras de energía.

La fachada del Proyecto Manubuild $-2^{a}$ generación de un proyecto iniciado por RLA

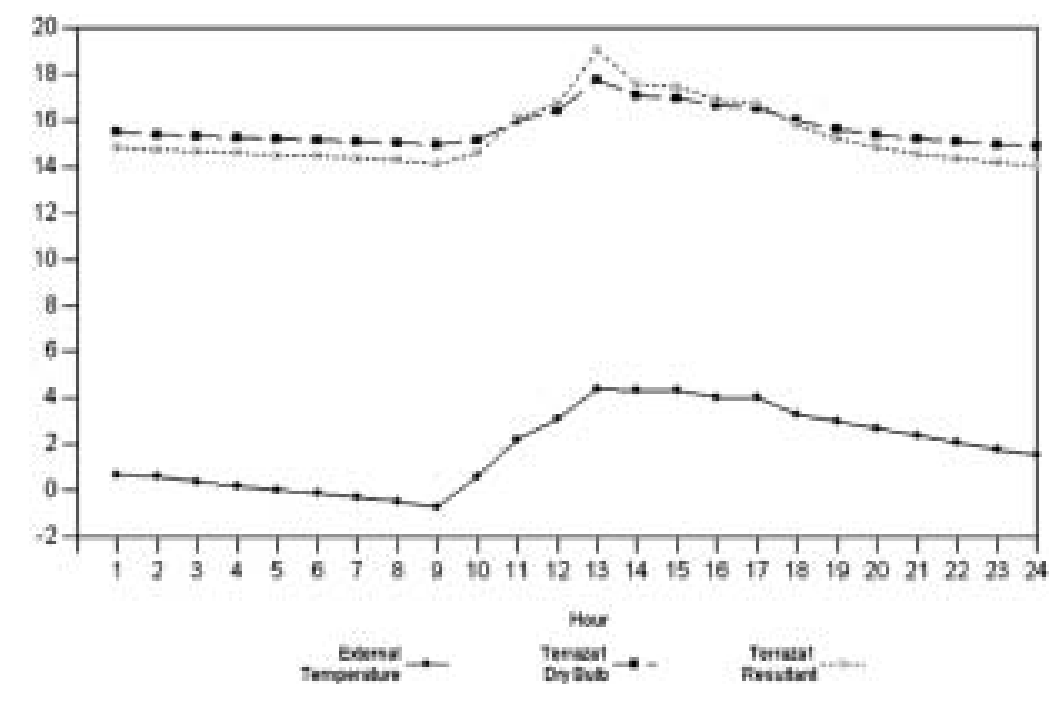

a partir del concurso para la nueva sede de la Agencia Andaluza de la Energía en SeviIla- permite dar una respuesta especializada a las diferentes solicitaciones funcionales del programa y energéticas de la climatología del lugar. Esta envolvente -desarrollada técnicamente junto a Sistemas TDM e Isofoton y comercializada por Bruesa Renovables- consiste en una doble piel que, aprovechando el comportamiento óptimo de las fachadas ventiladas frente a la radiación, está compuesta por diferentes módulos o píxeles que van formando la imagen energética y lo acondicionan en función del alcance económico que se desee alcanzar en la promoción
10.- La galería solar se cierra durante el invierno por un doble cerramientode vidrio que captura, por efecto invernadero, la radiación incidente durante los días soleados hasta elevar la temperatura del aire interior al entorno de los $20^{\circ} \mathrm{C}$ Para evitar el sobrecalentamiento de la galería durante el verano se han diseñado elementos de protección solar que rechazan la radiación directa (el gráfico corresponde a una simulación de la temperatura interior de la caldera solar en el caso más desfavorable). 

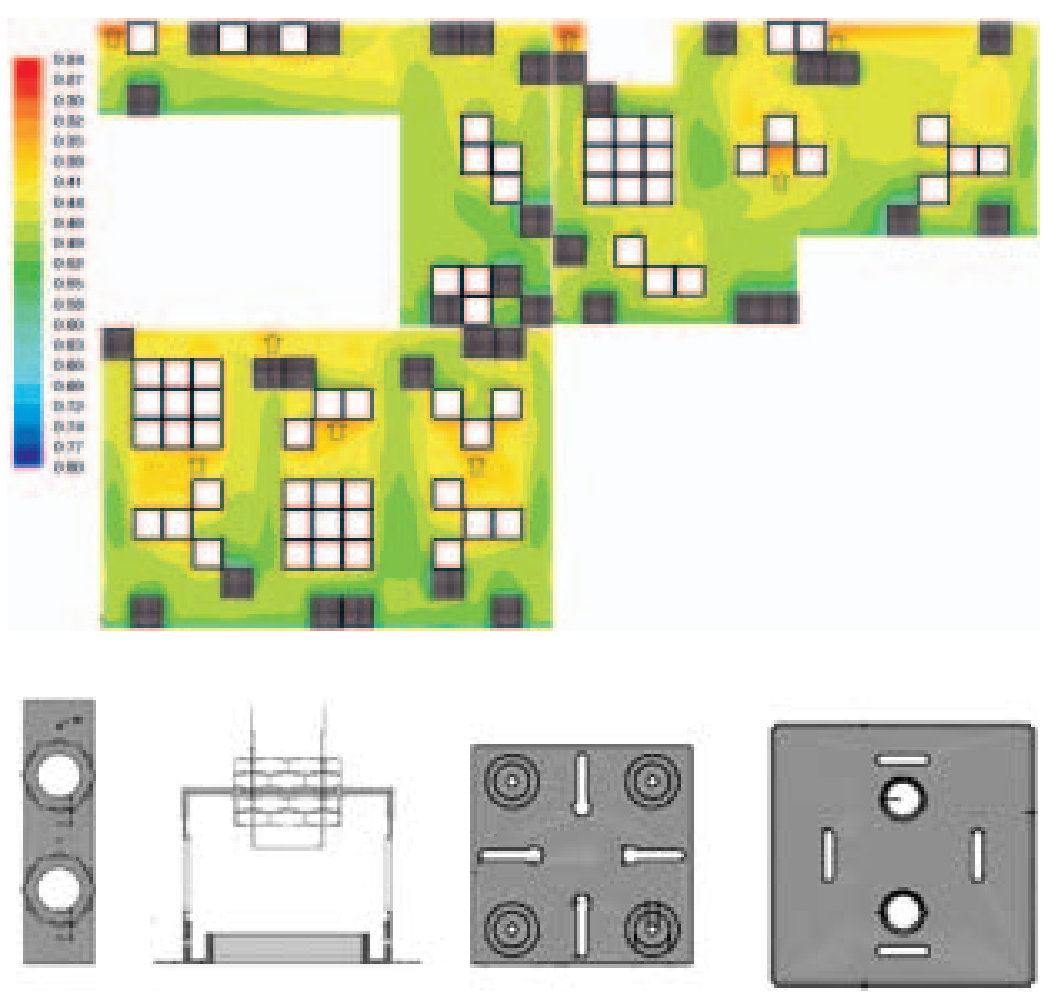

11.- Aprovechando las ventajas de la cámara ventilada frente a la radiación (ver simulación del comportamiento de la fachada con el programa Fluent), se ha diseñado una perfilaría especializada del comportamiento de acero galvanizado que puede sujetarse y anclarse de dos modos: bien a los cantos del forjado bien al paramento interior resistente: El resultado es una envolvente energética ventilada formada por un entramado universal de $1 \times 1 \mathrm{~m}$ donde pueden fijarse elementos pasivos o activos denominados "pixeles energéticos".

o, incluso, las preferencias de los usuarios. Se ha diseñado una perfilería especializada de acero galvanizado que puede sujetarse y anclarse de dos modos, bien a los cantos del forjado bien al paramento interior en el caso de que éste sea resistente. El resultado es una envolvente ventilada formada por un entramado universal de $1 \times 1 \mathrm{~m}$ donde pueden fijarse elementos pasivos o activos que hemos denominado "píxeles energéticos". Gracias a las experiencias de los aplacados "porosos" de los sistemas TDM, es posible, además, el sustituir los convencionales bastidores y los cuatro puntos de apoyo de los muros cortina por un sistema novedoso que ancla los píxeles a un único punto central, obteniendo así el máximo rendimiento con la mínima estructura.

Estos puntos de anclaje pueden convertirse, de este modo, también en los puntos de acceso a una sencilla estructura de canalizaciones que cumple con una doble función de anclaje y distribución de servicios. De esta manera, el catálogo de los píxeles está potencialmente abierto a cualquier función o material y puede ser, así, fotovoltaicos, termosolares, cerámicos, metálicos, etc., y

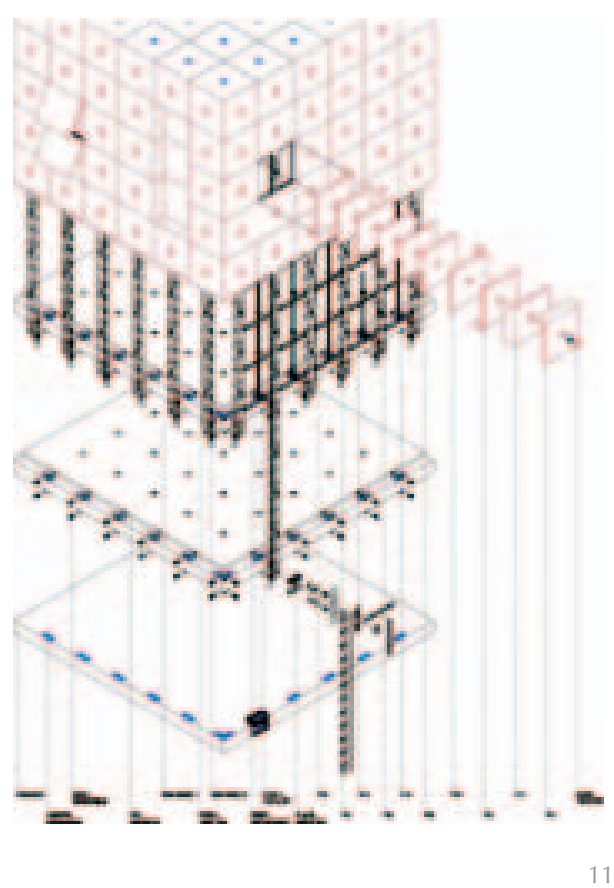

pueden disponerse en la fachada en función de los requisitos específicos que deban cumplir. De acuerdo a estas ideas, la fachada no es el resultado de una composición estética sino la respuesta a unas condiciones climáticas determinadas.

En el caso del solar objeto del proyecto, se estudió la capacidad térmica de la orientación Sur para elaborar el mapa energético del lugar.

De acuerdo a este mapa, se obtenían las directrices necesarias para situar cada píxel energético en el lugar más adecuado: píxeles fotovoltaicos en las áreas de mayor radiación, píxeles termosolares en las zonas de radiación media y píxeles cerámicos aislantes en las zonas con baja radiación.

Esta metodología, que analiza las componentes energéticas de cada lugar o clima, permite la respuesta más adecuada y específica a cada fachada, pero a través de un sistema constructivo industrializado universal, flexible y, por lo tanto, exportable a otras climatologías. Se trata de una experiencia compartible que origina un catálogo abierto y responde de manera directa a uno de los objetivos fundamentales del Proyecto Europeo Manubuild.
Cuadro 1. Características de los cerramientos

\begin{tabular}{|l|l|}
\hline Transmitancia de los muros & $0,66 \mathrm{~W} / \mathrm{m}^{2}{ }^{\circ} \mathrm{K}$ \\
\hline Transmitancia de suelos & $0,49 \mathrm{~W} / \mathrm{m}^{2}{ }^{\circ} \mathrm{K}$ \\
\hline Transmitancia de cubiertas & $0,38 \mathrm{~W} / \mathrm{m}^{2}{ }^{\circ} \mathrm{K}$ \\
\hline
\end{tabular}

*Balance energético de los sistemas aplicados
Cuadro 2. balance energético global ${ }^{*}$ 


\subsection{El Catálogo Manubuild}

El resultado del desarrollo de cada sistema específico, combinado con productos ya existentes en el mercado pero adaptados a los requerimientos particulares del proyecto, constituye un catálogo abierto de productos industrializados.

La compatibilidad entre estos sistemas es posible por el carácter geométricamente abierto de la matriz desarrollada en el proyecto (la red "Tres al Cubo") y los requerimientos de tolerancia exigidos para el correcto montaje de los componentes. Además, el hecho de que esta trama geométrica no se superponga al tipo edificatorio sino que lo genere permite alcanzar el máximo de flexibilidad espacial a las viviendas y el aprovechamiento óptimo de los ahorros energéticos derivados de una aplicación correcta de las estrategias bioclimáticas pasivas y los sistemas activos de generación de energía. El catálogo final desarrollado en el Proyecto Manubuild-cuyo listado resumido y clasificado por sistemas se adjunta a continuación- permitiría una

Cuadro 3. El "Catálogo Manubuild". Listado de sistemas industrializados abiertos

\begin{tabular}{|c|c|c|}
\hline Concepto & Sistema & Subsistema \\
\hline Estructura & $\begin{array}{l}\text { Estructura de acero } \\
\text { Estructura de hormigón prefabricado }\end{array}$ & $\begin{array}{l}\text { Vigas y pilares ejecutados en taller }+ \\
\text { placas alveolares } \\
\text { Arriostramientos mediante tensores } \\
\text { roscados } \\
\text { Placas alveolares } \\
\text { Muros de contención prefabricados }\end{array}$ \\
\hline Envolvente & $\begin{array}{l}\text { Cámara ventilada } \\
\text { Aislamientos ecológicos } \\
\text { Cerramiento prefabricado } \\
\text { Revestimientos exteriores (píxeles) }\end{array}$ & $\begin{array}{l}\text { Sistemas de anclaje } \\
\text { Manta de cáñamo } \\
\text { Paneles de hormigón } \\
\text { Píxeles cerámicos reflectantes } \\
\text { Píxeles vegetales } \\
\text { Píxeles fotovoltaicos } \\
\text { Píxeles captadores térmicos }\end{array}$ \\
\hline $\begin{array}{l}\text { Cerramientos y } \\
\text { Particiones }\end{array}$ & $\begin{array}{l}\text { Particiones entre viviendas } \\
\text { Particiones interiores } \\
\text { Cerramientos exteriores }\end{array}$ & $\begin{array}{l}\text { Aplacados cartón-yeso y composite de } \\
\text { hormigón } \\
\text { Aplacados cartón-yeso } \\
\text { Puertas escamoteables } \\
\text { Paneles prefabricados hormigón } \\
\text { Acristalamientos escamoteables } \\
\text { Parasol bioclimático } \\
\text { Vidrios baja emisividad }\end{array}$ \\
\hline Sistemas especiales & Módulo Corus & $\begin{array}{l}\text { Módulo prefabricado } \\
\text { completo de vivienda }\end{array}$ \\
\hline Instalaciones & $\begin{array}{l}\text { Aseos } \\
\text { Patinillos industrializados } \\
\text { Trazado instalaciones } \\
\text { Cuartos de instalaciones }\end{array}$ & $\begin{array}{l}\text { Módulos prefabricados aseo } \\
\text { Módulo DRAGADOS } \\
\text { Conductos prefabricados } \\
\text { Armarios prefabricados } \\
\text { Módulos prefabricados }\end{array}$ \\
\hline $\begin{array}{l}\text { Sistemas } \\
\text { bioclimáticos }\end{array}$ & $\begin{array}{l}\text { Sistema de reciclaje de agua } \\
\text { Cubierta }\end{array}$ & $\begin{array}{l}\text { Rejillas y aireadores } \\
\text { Serpentín de pretratamiento de aire } \\
\text { Patios de ventilación nocturna } \\
\text { Aljibes prefabricados } \\
\text { Cubierta ecológica }\end{array}$ \\
\hline & Zonas ajardinadas & Sistemas de recogida de agua de lluvia \\
\hline
\end{tabular}

12.- El catálogo final -abierto y perfectible- desarrollado en el Proyecto Manubuild permite una capacidad ilimitada de generar arquitectura mediante una combinatoria que haría posible la respuesta a cualquier climatología o requerimiento funcional. 
capacidad casi ilimitada de generar arquitectura mediante una combinatoria que haría posible la respuesta a cualquier climatología o requerimiento funcional.

\section{CONCLUSIONES}

- La estrategia de proyecto consiste en trabajar a partir de una matriz geométrica completamente abierta y capaz, por lo tanto, de asegurar desde el origen una compatibilidad con los productos y sistemas industrializados.
- Esta matriz geométrica determina, asimismo, el tipo edificatorio y favorece la integración de los sistemas activos y pasivos orientados al ahorro energético, de tal modo que el resultado espacial permite configuraciones flexibles no penalizadas por las servidumbres técnicas que lastran a la construcción convencional.

- El resultado del desarrollo del proyecto es un catálogo completamente abierto de productos y sistemas industrializados, susceptibles de ser utilizados para dar respuesta a cualquier climatología o requerimiento funcional.

\section{BIBLIOGRAFÍA}

(1) $\mathrm{a}+\mathrm{u}$ (ed.). 2005. "Structure \& Materials". Número especial. a + u, 412, Enero 2005: 7-167.

(2) Abalos, I.: "Técnica y arquitectura en la ciudad contemporánea". Ed. Nerea, Madrid, 1992.

(3) Addington, M.: 2007. "For smart materials, change is good". Architectural record, vol. 195, no 9, Septiempre 2007, pp. 160-165.

(4) Águila García, A. del: "La industrialización en los edificios de vivienda". Colegio oficial de arquitectos de Madrid, Madrid, 1984.

(5) Bensaude-Vincent, B.: 2000. "Eloge du mixte. Des composites aux matériaux intelligents". Techniques et architecture, no 448, Abril-Mayo, 2000, pp. 80-83.

(6) Kazi, A. S. (ed.): "Open Building Manufacturing. Core Concepts and Industrial Requirements". Manubuild, Finland, 2007.

(7) Martínez Calzón, J.: 2005.: "Los sistemas estructurales en la arquitectura contemporánea". Conferencia impartida en el Colegio Oficial de Arquitectos de Madrid el 21 de Febrero de 2005.

(8) Newby, F.: 1996. "The innovative uses of concrete by engineers and architects". Historic Concrete, Paper 11066.

(9) Ritter, A.: 2007. "Smart materials in architecture, interior architecture and design". Alemania: Birkhäuser.

(10) Sheehan, T.: 1995. "Advanced construction materials". The arcthitects' journal, 13 Julio, 1995, pp. $37-41$. 